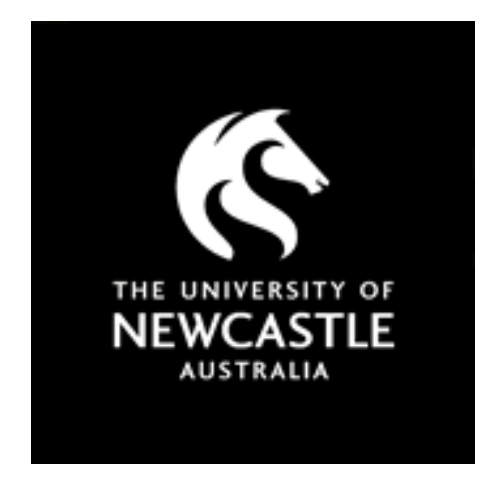

NOVA

University of Newcastle Research Online

nova.newcastle.edu.au

Isbister, Geoffrey K.; Heppell, Simon P.; Page, Colin B.; Ryan, Nicole M. “Adult clonidine overdose: prolonged bradycardia and central nervous system depression, but not severe toxicity", Published in Clinical Toxicology Vol. 55, Issue 3, p. 187-192. (2017)

Available from: $\underline{\text { http://dx.doi.org/10.1080/15563650.2016.1277234 }}$

This is an Accepted Manuscript of an article published by Taylor \& Francis Group in Clinical Toxicology on 20/01/2017, available online:

http://www.tandfonline.com/doi/full/10.1080/15563650.2016.1277234.

Accessed from: http://hdl.handle.net/1959.13/1352603 


\title{
Adult clonidine overdose: prolonged bradycardia and central nervous system depression, but not severe toxicity.
}

\begin{abstract}
Context: There are limited reports of adult clonidine overdose. We aimed to describe the clinical effects and treatment of clonidine overdose in adults.

Methods: This was a retrospective review of a prospective cohort of poisoned patients who took clonidine overdoses $(>200 \mu \mathrm{g})$. Demographic information, clinical effects, treatment, complications (central nervous system and cardiovascular effects), and length of stay (LOS) were extracted from a clinical database or medical records.
\end{abstract}

Results: From 133 admissions for clonidine poisoning (1988-2015), no medical record was available in 14 and 11 took staggered ingestions. Of 108 acute clonidine overdoses (median age $27 y$; 14-65y; 68 females), 40 were clonidine alone ingestions and 68 were clonidine with co-ingestants. Median dose taken was $2100 \mu \mathrm{g}$ (interquartile range [IQR]: 400-15,000 $\mu \mathrm{g}$ ). Median LOS was 21h (IQR:14-35h) and there were no deaths. Glasgow coma score [GCS] $<15$ occurred in $73 / 108(68 \%)$, and more patients taking co-ingestants $(8 / 68 ; 12 \%)$ had coma $(\mathrm{GCS}<9)$ compared to clonidine alone $(2 / 40 ; 5 \%)$. Miosis occurred in 31/108 $(29 \%)$ cases. Median minimum HR was 48bpm (IQR:40-57bpm), similar between clonidine alone and co-ingestant overdoses. There was a significant association between dose and minimum HR for clonidine alone overdoses $(\mathrm{p}=0.02) .82 / 108(76 \%)$ had bradycardia, median onset 2.5h post-ingestion (IQR:1.7-5.5h) and median duration $20 \mathrm{~h}(2.5-83 \mathrm{~h})$, similar for clonidine alone and co-ingestant overdoses. There were no arrhythmias. Three patients ingesting 8000$12000 \mu \mathrm{g}$ developed early hypertension. Median minimum systolic BP was $96 \mathrm{mmHg}$ (IQR:90-105mmHg) and hypotension occurred in 26/108 (24\%). 12/108 patients were intubated, but only 2 were clonidine alone cases. Treatments included activated charcoal (24), 
atropine (8) and naloxone (23). The median total naloxone dose was 2mg (IQR:1.2-2.4mg), but only one patient given naloxone was documented to respond with partial improvement in GCS.

Discussion: Clonidine causes persistent but not life-threatening clinical effects. Most patients develop mild central nervous system depression and bradycardia. Naloxone was not associated with improved outcomes.

Keywords: clonidine, poisoning, overdose, bradycardia, imidazoline 


\section{Introduction}

Clonidine has been available since the 1970's, initially for the treatment of hypertension, then opioid and alcohol dependence, sedation and more recently for the treatment of attention deficit disorder in children.[1,2] Clonidine is a 2-imidazoline derivative that was discovered in the course of testing it for use as a topical nasal decongestant. It is a centrally acting alpha2 adrenoreceptor agonist and an imidazoline receptor agonist, and these actions result in its central nervous system (CNS) depression and cardiovascular effects.[1]

Clonidine overdose is characterised by CNS depression, bradycardia and miosis, and can mimic opioid poisoning.[1, 3] Other clinical effects include early hypertension, followed by hypotension, hypothermia and respiratory depression.[1, 2, 4-8] Clonidine overdoses are uncommon, and the toxidromic triad of CNS depression, bradycardia and hypotension can often appear serious. In addition, the duration of some of the clinical effects may be prolonged, particularly the bradycardia. Case reports and reviews suggest clonidine may result in severe toxicity with ingestions of small amounts resulting in significant CNS depression and cardiovascular effects.[1, 7, 9]

Despite numerous case reports and series of paediatric clonidine ingestions, there are few reports of poisoning in adults,[9-11] and even fewer in the last 20 years since the widespread use of clonidine in addiction medicine. A lack of reported cases in adults and concerns about the potential severity of clonidine overdose has sometimes resulted in over-treatment, including both level of monitoring and use of antidotes in the authors' experience. It remains unclear whether the CNS depression and bradycardia are always of sufficient severity to require interventions with either antidotes or intubation and ventilation. For this reason further information on the time course and severity of adult clonidine ingestions is required. 
A number of antidotes have been used for the treatment of clonidine overdose, including naloxone and atropine with variable success. There continues to be controversy over the role of naloxone and whether it reverses coma or influences cardiovascular effects.[3] Atropine is often used to treat the bradycardia but again there is limited evidence to decide if this is required, considering the short duration of action of atropine compared to clonidine. It is important to determine whether specific treatments are beneficial in clonidine poisoning, or whether supportive care alone is sufficient.[12]

This study aimed to determine the severity and duration of clinical effects resulting from clonidine overdose in adults. In addition we report the effect of various treatments in clonidine poisoning to attempt to improve our understanding of different interventions. 


\section{Methods}

\section{Study design and setting:}

We undertook a retrospective observational study of adult clonidine overdoses admitted to an inpatient toxicology unit. The toxicology unit admits all adult ( $>15$ years age) poisoning and overdose presentations to the emergency department from a population of over 500,000 people. All admissions to the unit have demographic and clinical information collected using a standardised data collection form, which is part of the emergency department assessment. Further information on complications, outcomes and treatment is collected throughout the admission, and all cases are reviewed and additional information obtained from the admitting clinical toxicologist. Trained research assistants enter all the data into a relational database within a week of the presentation to hospital. Toxicology patients are seen daily by the toxicology unit and treatment is according to the attending clinical toxicologist. The use of the database and patient medical records for research has been granted by the Area Health Service Human Research Ethics Committee.

\section{Patients}

The toxicology database was searched from January 1987 to September 2015 for all adult (>15y) clonidine overdose or poisoning admissions to the toxicology unit. Ingestion of clonidine was based on the history from the patient, collateral history from family and friends, and any information recorded by ambulance or emergency services. Overdoses were included with and without coingestants. Ingestions $<200 \mu$ g were excluded. Staggered ingestions were included but reported separately to acute overdoses.

\section{Data Collection}


All data for clonidine overdoses was extracted from the toxicology database. Medical records were reviewed for missing data and for information on duration of the bradycardia and hypotension, and response to antidote treatment. Data extracted included: demographic information (sex, age), ingestion details (dose, time of ingestion, acute or staggered ingestion, and coingestants), medication history, clinical observations (heart rate [HR] - minimum, onset and duration of bradycardia; blood pressure $[\mathrm{BP}]$ - maximum, minimum and duration of hypotension; arrhythmias; Glasgow coma score [GCS]; miosis; temperature - minimum), treatment (charcoal, admission to intensive care unit [ICU]; intubation/mechanical ventilation; and antidotes - atropine, naloxone and inotropes) and length of stay (LOS). For each parameter the most extreme appropriate measurement (maximum or minimum) was recorded from the observations.

\section{Outcomes}

Pre-defined outcomes included: bradycardia (HR < 60 beats per minute [bpm]), hypotension (systolic BP < 90mmHg), hypertension (systolic BP > 180mmHg), coma $(\mathrm{GCS}<$ 9), hypothermia (temperature $<35^{\circ} \mathrm{C}$ ), arrhythmia and death. The duration of bradycardia and hypotension was recorded from the time of onset (or first recording) until resolution or discharge. The administration of antidotes and reported response to antidotes was recorded as a treatment outcome.

\section{Analysis}

Dichotomous outcomes/variables were reported with 95\% confidence intervals (CI) which were calculated using Wilson's procedure and continuity correction. Continuous variables were reported as medians, interquartile ranges (IQR) and ranges where appropriate. Linear regression of minimum $\mathrm{HR}$, minimum $\mathrm{BP}$ and minimum GCS versus dose was used to determine if there were any associations. All statistical analysis was undertaken in Graphpad 
Prism, version 6.05 for Windows. (GraphPad Software, La Jolla California CA, U.S.A.; www.graphpad.com). 


\section{Results}

There were 133 admissions for clonidine overdose or poisoning between January 1988 and September 2015. Medical records were not available for 14 cases and 11 took staggered ingestions, leaving 108 acute ingestions. In 40/108, clonidine was ingested alone. In the remaining 68 there were one to five co-ingestants. The commonest co-ingestants were benzodiazepines, antipsychotics, opioids, anticonvulsants and alcohol (Supplementary Table 1). Table 1 compares cases with clonidine alone to those with co-ingestants. Sixty eight of the $108(63 \%)$ were female, and the median age of patients was 27 years (range: 14 to 65 years). The source of the medication was the patient's in 78 cases, someone else's medication in 24 cases (children [12], other family member [6], other person [6]), and the source was unknown in six cases. The median dose taken was $2100 \mu \mathrm{g}(400-15,000 \mu \mathrm{g})$ which did not differ between clonidine alone cases and those with coingestants. The median length of stay was $21 \mathrm{~h}$ (interquartile range: 14 to $35 \mathrm{~h}$ ) which did not differ for patients taking coingestants. There were no deaths.

\section{Clinical Effects}

The median minimum GCS was 14 (IQR: 13 to 15). There was a decreased level of consciousness (GCS < 15) in 73/108 (68\%) of admissions, and coma in $10(9 \%)$. There were more patients with severe CNS depression (coma or GCS < 9) in the group co-ingesting medications $(8 / 68 ; 12 \%)$ compared to those ingesting clonidine alone $(2 / 40 ; 5 \%)$. All eight patients with coma co-ingested a benzodiazepine: one with only a benzodiazepine, two with two different benzodiazepines, two with heroin, two with alcohol and one with chlorpromazine and methadone. There was no association between the dose in 40 clonidine alone overdoses and minimum GCS ( $\mathrm{p}=0.40)$. Miosis occurred in 31 (29\%) admissions and hypothermia in 11 patients. 
The median minimum HR for all admissions was $48 \mathrm{bpm}$ (IQR: 40 to $57 \mathrm{bpm}$; range: 32 to $88 \mathrm{bpm})$, which was similar between the clonidine alone group and the group co-ingesting other medications. There was a significant association between dose and minimum HR in the clonidine alone group and the data was fitted using linear regression (slope $=-0.002 \pm 0.001$ $\mathrm{bpm} / \mu \mathrm{g}, \mathrm{p}=0.02$; Figure 1). Eighty two of $108(76 \%)$ patients developed sinus bradycardia with a median onset of $2.5 \mathrm{~h}$ post-ingestion (IQR: 1.7 to $5.5 \mathrm{~h} ; \mathrm{N}=76$ ). The onset of bradycardia was similar for clonidine alone and patients co-ingesting other medications (Figure 2A). Bradycardia persisted for a median of 20h (2.5 to $83 \mathrm{~h} ; \mathrm{N}=76$; Figure 2A) and was similar for clonidine alone and mixed ingestions. Figure $2 \mathrm{~B}$ shows the proportion recovered to a normal HR over time showing no difference between clonidine alone and coingestants. Thirty four of the 82 patients $(41 \%)$ with sinus bradycardia were discharged still bradycardic. No other arrhythmias were reported.

Three patients ingesting large amounts $(8000,10000$ and $12000 \mu \mathrm{g})$ developed early hypertension. In two patients the BP decreased over $2 \mathrm{~h}$ and they then developed mild hypotension (minimum systolic BP $78 \mathrm{mmHg}$ and $84 \mathrm{mmHg}$ ).

The median minimum systolic BP was $96 \mathrm{mmHg}$ (IQR: 90 to $105 \mathrm{mmHg}$; range: 70 to $154 \mathrm{mmHg}$ ), and there was no relationship between dose and minimum systolic BP for clonidine alone and co-ingestant overdoses. Hypotension occurred in 26 of 108 (24\%) and had a median onset of 4.2h (IQR: 2.to 7.8h) in clonidine alone overdoses and a median onset of $8 \mathrm{~h}$ (IQR: 3.7 to $18 \mathrm{~h}$ ) in clonidine overdoses with co-ingestants (Figure 3). The duration of hypotension was $11 \mathrm{~h}$ (IQR: 1.8 to $13 \mathrm{~h}$ ) in clonidine alone overdoses and 2.8h (IQR: 1.7 to 6h) in clonidine overdoses with co-ingestants (Figure 3).

\section{Treatment}


Twenty six patients were admitted to ICU and twelve were intubated and ventilated. Two patients who ingested clonidine alone were intubated. One patient was intubated for aggression. The second ingested $5000 \mu \mathrm{g}$ and was intubated in a peripheral hospital for decreased level of consciousness prior to retrieval. The remaining 10 intubated patients ingested other sedative drugs and included six patients with coma (GCS < 9).

Treatments included activated charcoal (24), atropine (8) and naloxone (23). The median total dose of naloxone administered was $2 \mathrm{mg}$ (IQR: 1.2 to $2.4 \mathrm{mg}$; Range: 0.1 to $14 \mathrm{mg}$ ). Twenty two of the 23 patients given naloxone were not reported to respond by the treating clinician (no change in GCS and no change in HR). One patient was documented to have a partial improvement in GCS, but had also recently injected heroin. Patients administered naloxone

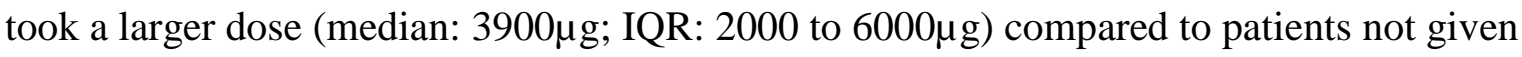

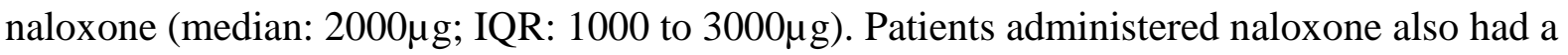
lower minimum GCS [median 9 (IQR: 5 to 14) vs. 14 (IQR: 14 to 15)] and had a longer LOS [median 4lh (IQR: 19 to 60h) vs. 19h (IQR: 12 to 27h)], compared to those not given naloxone.

Atropine was given on one or more occasions in eight patients. In five patients there was evidence on the observation chart of a transient increase in the HR, which only lasted for $1 \mathrm{~h}$. In one patient the increase in HR was also associated with an increase in BP. One patient was given adrenaline for hypotension by the retrieval team, which was associated paradoxically with worsening hypotension.

\section{Staggered Ingestions}

There were eleven patients (seven males; median age 34y [19 to 42y]) who took staggered ingestions over a median period of 1 day ( 0.5 to 7 days). The median ingested dose was $3600 \mu \mathrm{g}(1500$ to $30,000 \mu \mathrm{g})$ and ten took co-ingestants, most commonly benzodiazepines. 
Ten developed bradycardia, three had hypotension, four had a GCS $<15$ and none had a GCS <9. The median LOS was 22 (IQR: 12 to $42 \mathrm{hr}$ ) and one was admitted to ICU for cardiac monitoring only. Atropine was used in one and naloxone in two with no reported benefit. 


\section{Discussion}

Our study confirms the previously reported clinical effects of clonidine poisoning in adults, but shows that these are not usually severe and complications are rare. Bradycardia appears to persist on average for almost a day and may have contributed to the longer LOS compared to the median LOS for all overdose admissions to the same unit.[13] Respiratory interventions for CNS depression were uncommon in clonidine alone overdoses and were more common in patients coingesting other medications. Both naloxone and atropine were used in a small number of cases but did not appear to result in major improvement in the patients' condition.

The study defines the severity, variability and duration of bradycardia and hypotension in clonidine poisoning. Bradycardia was significantly associated with the dose ingested in the clonidine alone overdoses (Figure 1), and developed within hours of ingestion in both clonidine alone and clonidine with co-ingestant overdoses (Figure 2A). Bradycardia lasted for 24 to $48 \mathrm{~h}$ (Figure $2 \mathrm{~A}$ ) and was the same for clonidine alone and clonidine with co-ingestant overdoses. In contrast, hypotension was not dose-related, had a later and far more variable onset and variable duration (Figure 2). The onset and duration of hypotension was also different between clonidine alone and clonidine with co-ingestant overdoses. This possibly suggests that the bradycardia is a direct effect of clonidine (imidazoline or alphaadrenoceptor agonism) compared to the hypotension, which is more likely to be multifactorial, including being secondary to bradycardia, and being influenced by coingestants. Only one patient was treated with inotropes for hypotension, which was associated with paradoxical worsening of the hypotension and bradycardia.

Two common questions asked in regards to clonidine overdose are 1) Do they require ICU or high dependency unit admission? and 2) How long should they be observed? Based on the fact that only two clonidine alone overdoses developed coma and only two required 
intubation, the majority of clonidine alone overdoses could be observed in an emergency short stay unit with standard HR and oxygen saturation monitoring. In patients co-ingesting clonidine with other medications, admission to ICU may be more dependent on the coingestants. Almost half of the bradycardic patients were discharged with bradycardia, which means that the duration is under-estimated in this study. In addition, the absence of other arrhythmias and much shorter duration of hypotension suggests that the bradycardia is relatively benign. Patients who are normotensive, alert and are not symptomatic (dizzy) after $24 \mathrm{~h}$ are most likely safe for discharge.

There are few studies of adult clonidine overdose. The earliest and only relatively large case series is from Stein et al. who reported 37 adult overdoses from the National Poison Information Service in London.[9] The range of doses appeared to be similar to our study and they reported a similar constellation of clinical effects, with more CNS depression and less bradycardia.[9] Other smaller studies that include adult patients report similar effects but less information on duration and treatments.[10, 11, 14] These earlier studies all report more severe poisoning with higher rates of CNS depression, respiratory depression and cardiac conduction abnormalities. These studies also suggest that the frequency of CNS depression and bradycardia is less common in adults compared to children. However, in comparing our study and more recent paediatric studies, the frequency of clinical effects and severity appears to be similar.[2]

Limitations to the study were the retrospective design, possible inaccuracies in the ingested dose and reported drugs not confirmed by laboratory analysis. The majority of the data used in the study was recorded prospectively within a week of the patient admission, with pre-defined outcomes based on the pre-formatted admission sheets. However, some of the data including the time course of the clinical effects and response to treatment were extracted from the medical 
record. These latter outcomes are limited by how well the physicians or nursing staff recorded information.

An important limitation of the study was that interventions were not randomised, but determined by the admitting clinical toxicologist or critical care physician. There are likely to be confounding influences on the patient groups given particular treatments. However, the almost complete absence of reports of responses to naloxone provides some support that naloxone is not clinically effective. In addition, naloxone appeared to be administered to the more severely poisoned patients, who are most likely to benefit, based on these patients taking larger doses, having lower GCS and longer LOS; naloxone itself is unlikely to double the LOS.

Confirmation of whether clonidine was actually ingested could not be confirmed analytically and the clonidine dose was based on patient report. Although this is commonly thought to be a limitation, we have reported on numerous occasions the accuracy of the patient history, including the reported dose.[15] The association between dose and minimum HR supports this, similar to other studies from the same toxicology unit.[16, 17]

Our study found that clonidine causes persistent but not life threatening clinical effects including bradycardia and CNS depression. Although these initially appear to be clinically significant, they are unlikely to predict a poor patient outcome. The majority of patients will develop mild CNS depression and bradycardia which could be safely observed in an emergency short stay ward. The study did not find support for the routine use of either naloxone or atropine, but atropine may be required in patients with bradycardia and significant hypotension. 



\section{References}

1. Lowry JA, Brown JT. Significance of the imidazoline receptors in toxicology. Clin Toxicol (Phila) 2014; 52: 454-69.

2. Sinha Y, Cranswick NE. Clonidine poisoning in children: a recent experience. $J$ Paediatr Child Health 2004; 40: 678-80.

3. Seger DL. Clonidine toxicity revisited. J Toxicol Clin Toxicol 2002; 40: 145-55.

4. Spiller HA, Colvin JM, Villalobos D, Johnson PB, Anderson DL, Klein-Schwartz W. Clonidine ingestion in children. Journal of Toxicology: Clinical Toxicology 2003; 41: 663.

5. Klein-Schwartz W. Trends and toxic effects from pediatric clonidine exposures. Archives of Pediatrics and Adolescent Medicine 2002; 156: 392-6.

6. Nichols MH, King WD, James LP. Clonidine poisoning in Jefferson County, Alabama. Annals of Emergency Medicine 1997; 29: 511-7.

7. Wiley JF, 2nd, Wiley CC, Torrey SB, Henretig FM. Clonidine poisoning in young children. J Pediatr 1990; 116: 654-8.

8. Bamshad MJ, Wasserman GS. Pediatric clonidine intoxications. Vet Hum Toxicol 1990; 32: 220-3.

9. Stein B, Volans GN. Dixarit overdose: the problem of attractive tablets. $\mathrm{Br} \mathrm{Med} \mathrm{J}$ 1978; $2: 667-8$.

10. Gossinger H, Hruby K, Haubenstock A, Jung M, Pfister R. [Poisoning with clonidin hydrochloride in children and adults]. Wien Klin Wochenschr 1983; 95: 232-5.

11. Anderson RJ, Hart GR, Crumpler CP, Lerman MJ. Clonidine overdose: report of six cases and review of the literature. Ann Emerg Med 1981; 10: 107-12.

12. Isbister GK, Buckley NA. Therapeutics in clinical toxicology: in the absence of strong evidence how do we choose between antidotes, supportive care and masterful inactivity. $\mathrm{Br} J$ Clin Pharmacol 2016; 81: 408-11. 
13. Buckley NA, Whyte IM, Dawson AH, Isbister GK. A prospective cohort study of trends in self-poisoning, Newcastle, Australia, 1987-2012: plus ca change, plus c'est la meme chose. Med J Aust 2015; 202: 438-42.

14. Conner CS, Watanabe AS. Clonidine overdose: a review. Am J Hosp Pharm 1979; 36: 906-11.

15. Isbister GK, Friberg LE, Stokes B, Buckley NA, Lee C, Gunja N, Brown SG, MacDonald E, Graudins A, Holdgate A, Duffull SB. Activated charcoal decreases the risk of QT prolongation after citalopram overdose. AnnEmerg Med 2007; 50: 593-600, .

16. McNamara K, Isbister GK. Hyperlactataemia and clinical severity of acute metformin overdose. Intern Med J 2015; 45: 402-8.

17. Cooper JM, Brown JA, Cairns R, Isbister GK. Desvenlafaxine overdose and the occurrence of serotonin toxicity, seizures and cardiovascular effects. Clin Toxicol (Phila) $2016 ; 1-7$. 
Table 1: Demographic information, clinical effects, outcomes and treatments for clonidine overdoses comparing clonidine alone to clonidine with coingestant overdoses.

\begin{tabular}{|c|c|c|c|c|}
\hline Clinical Effect & Alone (40) & $\%$ & Coingestant (68) & $\%$ \\
\hline Female & 23 & $58 \%$ & 45 & $66 \%$ \\
\hline Age, y; Median (IQR) & $26(14$ to 55$)$ & & $27(16$ to 65$)$ & \\
\hline Dose, $\mu \mathrm{g} ;$ Median (IQR) & $2175(1313-3500)$ & & $\begin{array}{c}2000(1025- \\
3375)\end{array}$ & \\
\hline \multicolumn{5}{|l|}{ Cardiovascular Effects } \\
\hline $\begin{array}{r}\text { Bradycardia }(\text { HR }<60) \\
\text { Median minimum HR; Median }\end{array}$ & $\begin{array}{c}27 \\
48(40-62)\end{array}$ & $68 \%$ & $\begin{array}{c}55 \\
47(40-55)\end{array}$ & $81 \%$ \\
\hline Hypotension (Sys BP<90mmHg) & 10 & $25 \%$ & 16 & $24 \%$ \\
\hline $\begin{array}{r}\text { Severe Hypertension (Sys } \\
\text { BP }>180 \mathrm{mmHg})\end{array}$ & 0 & $0 \%$ & 2 & $3 \%$ \\
\hline \multicolumn{5}{|l|}{ Central Nervous System Effects } \\
\hline GCS $<15$ & 22 & $55 \%$ & 51 & $75 \%$ \\
\hline Coma $($ GCS $<9)$ & 2 & $5 \%$ & 8 & $12 \%$ \\
\hline Miosis & 10 & $25 \%$ & 21 & $31 \%$ \\
\hline \multicolumn{5}{|l|}{ Hospital Admission } \\
\hline Length of Stay, h; Median (IQR) & $21(11$ to 27$)$ & & $20(15$ to 35$)$ & \\
\hline Intensive Care & 9 & $23 \%$ & 17 & $25 \%$ \\
\hline Intubated & 2 & $5 \%$ & 10 & $15 \%$ \\
\hline Treatments & & & & \\
\hline
\end{tabular}




\begin{tabular}{|r||c|c||c|c|}
\hline Atropine & 4 & $23 \%$ & 4 & $6 \%$ \\
\hline Naloxone & 7 & $18 \%$ & 16 & $24 \%$ \\
\cline { 2 - 5 } & & & & \\
\hline Activated Charcoal & 10 & $25 \%$ & 14 & $21 \%$ \\
\hline
\end{tabular}




\section{Figure Legends}

Figure 1: Relationship between the dose and minimum heart rate for clonidine alone overdoses (filled circles) and clonidine overdoses with co-ingestants (open circles) demonstrating a significant association between dose and decreasing heart rate for clonidine alone overdoses $(\mathrm{p}=0.022)$.

Figure 2: Scatter plots of the time of onset from ingestion to bradycardia for clonidine alone (median 2.5h; IQR: 1.6 to 5.8h) and co-ingestant overdoses (median 2.5h; IQR: 1.8 to $5.5 \mathrm{~h}$ ), and the duration of bradycardia for clonidine alone overdoses (median 22h; IQR: 14 to 39h) and clonidine with co-ingestant overdoses (median 20h; IQR: 12 to 31h) [A], and the time to recovery of heart rate to normal for clonidine alone and clonidine with co-ingestant overdoses [B].

Figure 3: Scatter plots of the time of onset from ingestion to hypotension for clonidine alone (median 4.2h; IQR: 2.2 to $7.8 \mathrm{~h}$ ) and co-ingestant overdoses (median 8h; IQR: 3.7 to $18 \mathrm{~h}$ ) and duration of hypotension for clonidine alone (median 11h; IQR: 1.8 to $13 \mathrm{~h}$ ) and co-ingestant overdoses (median 2.8h; IQR: 1.7 to $6 \mathrm{~h}$ ). 